\title{
EXTREMAL SOLUTIONS TO A CLASS OF MULTIVALUED INTEGRAL EQUATIONS IN BANACH SPACE ${ }^{1}$
}

\author{
SERGIU AIZICOVICI ${ }^{2}$ \\ Ohio University \\ Department of Mathematics \\ Athens, $O H$ 45701, USA \\ and \\ NIKOLAOS S. PAPAGEORGIOU \\ Florida Institute of Technology \\ Department of Applied Mathematics \\ Melbourne, FL 32901, USA
}

\begin{abstract}
We consider a nonlinear Volterra integral inclusion in a Banach space. We establish the existence of extremal integral solutions, and we show that they are dense in the solution set of the original equation. As an important application, we obtain a "bang-bang" theorem for a class of nonlinear, infinite dimensional control systems.
\end{abstract}

Key words: $m$-accretive operator, Volterra integral inclusion, integral solution, "bang-bang" principle.

AMS (MOS) subject classifications: $\quad 45 \mathrm{~N} 05,34 \mathrm{G} 20,45 \mathrm{D} 05$.

\section{INTRODUCTION}

In a recent paper [2], we discussed the existence and properties of integral solutions to the multivalued Volterra equation

$$
x(t)+\int_{0}^{t} k(t-s)(A x(s)+F(s, x(s))) d s \ni g(t), t \in T=[0, b]
$$

in a Banach space $X$. In $(\underline{1}), A: D(A) \subseteq X \rightarrow 2^{X}$ is an $m$-accretive operator, $F: T \times X \rightarrow 2^{X},\{\emptyset\}$ is a closed valued perturbation, $k: T \rightarrow R$ and $g: T \rightarrow X$.

The current note is concerned with the nonlinear Volterra integral inclusion

\footnotetext{
${ }^{1}$ Received: March, 1992. Revised: June, 1992.

${ }^{2}$ This author was supported in part by the National Science Foundation under grant DMS-91-11794.
} 


$$
x(t)+\int_{0}^{t} k(t-s)(A x(s)+\operatorname{ext} F(s, x(s))) d s \ni g(t), t \in T=[0, b]
$$

where $\operatorname{ext} F(s, x(s))$ denotes the set of extremal points of $F(s, x(s))$. Equations of this form arise in the study of nonlinear distributed parameter control system (in particular, the derivation of "bang-bang" principles, cf. [3]), as well as in the description of obstacle problems [9].

We remark that the theory developed in [2] for equation (1) can no longer be applied to $(\underline{\underline{2}})$, since the multifunction $(t, x) \rightarrow \operatorname{ext} F(t, x)$ is not necessarily closed valued and, in general, we cannot say anything about its continuity properties. From an abstract viewpoint, the present study is a direct attempt to extend some of the existence and relaxation theorems of [2] to equation (2) (cf. [2, theorems 3.2, 3.4 and 4.1]). At the same time, we generalize in various directions earlier results for Volterra integral and integro-differential inclusions $[1,3,11,16,18$, $20,22]$, and multivalued differential equations $[14,17,21,24]$. (It is worth mentioning that in the case when $k \equiv 1$, equations $(\underline{\underline{1}})$ and $(\underline{\underline{2}})$ are formally equivalent to a nonlinear evolution inclusion in $X$ ).

The plan of the paper is as follows. Section 2 contains some background material on multifunctions, $m$-accretive operators, and abstract Volterra equations. Section 3 is concerned with the existence of integral solutions to equation (2). In Section 4, we show that every integral solution of $(\underline{\underline{1}})$ can be approximated, in the $C(T, X)$ norm, by solutions of (2). An example of an integro-partial differential equation is also discussed. In the last section, we use our abstract results to derive "bang-bang" type theorems for nonlinear infinite dimensional control systems.

\section{PRELIMINARIES}

Throughout this paper, $T$ denotes the interval $[0, b]$ (endowed with the Lebesgue measure) and $X$ stands for a real separable Banach space of norm $\|\cdot\|$. The following notation will also be used: $P_{f(c)}(X)=\{B \subseteq X: B$ is nonempty, closed, (convex) $\}$, $P_{w k(c)}(X)=\{B \subseteq X: B$ is nonempty, weakly-compact, (convex) $\}$.

A multifunction $F: T \rightarrow P_{f}(X)$ is said to be measurable if for every $x \in X$, the $\mathbb{R}_{+}$ valued function $t \rightarrow d(x, F(t))=\inf \{\|x-z\|: z \in F(t)\}$ is measurable. By $S_{F}^{1}$ we will denote the set of $L^{1}(T, X)$ selectors of $F$, namely $S_{F}^{1}=\left\{f \in L^{1}(T, X): f(t) \in F(t)\right.$ a.e. $\}$. This set may be empty. For a measurable multifunction $F, S_{F}^{1}$ is nonempty if and only if $t \rightarrow \inf \{\|x\|: x \in F(t)\} \in L^{1}\left(T, \mathbb{R}_{+}\right)$. Furthermore, $S_{F}^{1}$ is a decomposable subset of $L^{1}(T, X)$, 
i.e., if $f_{1}, f_{2} \in S_{F}^{1}$ and $B$ is a Borel subset of $T$, then $\chi_{B} f_{1}+\chi_{B} c f_{2} \in S_{F}^{1}$, where $\chi_{B}$ denotes the characteristic function of $B$.

On $P_{f}(X)$ we can define a generalized metric, known in the literature as the Hausdorff metric, by

$$
h(B, C)=\max \left[\sup _{b \in B} d(b, C), \sup _{c \in C} d(c, B)\right]
$$

for all $B, C \in P_{f}(X)$. The space $\left(P_{f}(X), h\right)$ is complete. A multifunction $G: X \rightarrow P_{f}(X)$ is said to be Hausdorff continuous ( $h$-continuous), if it is continuous from $X$ into the metric space $\left(P_{f}(X), h\right)$.

If $Y, Z$ are Hausdorff topological spaces, a multifunction $H: Y \rightarrow 2^{Z} \cdot\{\emptyset\}$ is lower semicontinuous (l.s.c.) if for every closed set $C$ in $Z$, the set $H^{+}(C)=\{y \in Y: H(y) \subseteq C\}$ is closed in $Y$.

The remainder of this section is devoted to a brief discussion of $m$-accretive operators and Volterra equations in Banach spaces. For more details we refer the reader to [5, 7, 11, 24].

Let $A$ be a set-valued operator in $X$ with domain $D(A)$. We say that $A$ is accretive if $\left\|x_{1}-x_{2}\right\| \leq\left\|x_{1}-x_{2}+\lambda\left(y_{1}-y_{2}\right)\right\|$, for all $\lambda>0$ and all $y_{i} \in A x_{i}, i=1,2$. If in addition, $I+\lambda A$ is surjective for all (equivalently, some) $\lambda>0$, where $I$ stands for the identity operator on $X$, then $A$ is called $m$-accretive. If $A$ is $m$-accretive, then $-A$ generates a contraction semigroup $S(t), t \geq 0$ on $\overline{D(A)}$. We will say that the semigroup generated by $-A$ is compact, if $S(t)$ is a compact operator for each $t>0$.

We next consider the Volterra integral equations $(\underline{\underline{1}})$ and $(\underline{\underline{2}})$, and assume that $A$ is $m$ accretive, $F: T \times X \rightarrow 2^{X}\{\{\}, \quad k \in A C(T, \boldsymbol{R})$ such that $\dot{k} \in B V(T, \boldsymbol{R})$ and $k(0)=1$, and $g \in W^{1,1}(T, X)$ with $g(0) \in \overline{D(A)}$. Following [11], we define $G: C(T, \overline{D(A)}) \rightarrow L^{1}(T, X)$ and $x_{0} \in \overline{D(A)}$ by

(i) $\quad G(x)(\cdot)=h(\cdot)+r * h(\cdot)-r(0) x(\cdot)+r(\cdot) x_{0}-x * \dot{r}(\cdot)$, with $x * \dot{r}(t)=\int_{0}^{t} x(t-s) d r(s)$

(ii) $h(t)=\dot{g}(t)$

(iii) $x_{0}=g(0)$,

(iv) $\quad r+a * r=-a, a=\dot{k}$.

By an integral solution of equation (1) (resp. (2)), we mean a function $x \in C(T, X)$ such that there exists $f \in L^{1}(T, X)$ with $f(t) \in F(t, x(t))$, a.e. (resp. $f(t) \in \operatorname{ext} F(t, x(t)$ ), a.e.), and $x$ is an integral solution (in the sense of Benilan [7]) of the initial-value problem $\dot{x}(t)+A x(t) \ni G(x)(t)-f(t), x(0)=x_{0}$, where $G$ and $x_{0}$ are given by $(\underline{3})$. 


\section{AN EXISTENCE THEOREM}

We begin this section with a simple lemma that will frequently be used in the sequel. Let $L_{w}(T, X)$ denote the space of equivalence classes of Bochner integrable functions $x: T \rightarrow X$, with the ("weak") norm $\|x\|_{w}=\sup \left\{\left\|\int_{t_{1}}^{t_{2}} x(s) d s\right\|: 0 \leq t_{1} \leq t_{2} \leq b\right\}$. The notation $\stackrel{\|\cdot\|_{w}}{\rightarrow}$ stands for convergence in $L_{w}(T, X)$.

Lemma 3.1: Assume that $X^{*}$ has the Radon-Nikodym property. Let $\left\{f_{n}\right\}_{n \geq 1} \subseteq L^{p}(T, X), 1<p<\infty$ be such that $\sup _{n \geq 1}\left\|f_{n}\right\|_{p}<\infty$ and $f_{n} \stackrel{\|\cdot\|}{\rightarrow} w_{0}$, as $n \rightarrow \infty$. Then $f_{n} \rightarrow 0$, weakly in $L^{p}(T, X)$.

Proof: $\quad$ By $[12, \quad$ theorem $1, \quad$ p. $\quad 98], \quad\left(L^{p}(T, X)\right)^{*}=L^{q}\left(T, X^{*}\right), \quad$ where $p^{-1}+q^{-1}=1$. Let $((\cdot, \cdot))$ denote the duality pairing between $L^{p}(T, X)$ and $L^{q}\left(T, X^{*}\right)$. Since $\left\{f_{n}\right\}$ is bounded in $L^{q}(T, X)$, by assumption, and the space of $X^{*}$-valued simple functions on $T$ is dense in $L^{q}\left(T, X^{*}\right)$, we only need to show that $\left(\left(f_{n}, s\right)\right) \rightarrow 0$, as $n \rightarrow \infty$, for each $s: T \rightarrow X^{*}$ of the form $s(t)=\sum_{k=1}^{N} \chi_{\left(t_{k-1}, t_{k}\right)}(t) v_{k}^{*}$, with $v_{k}^{*} \in X^{*}$. We have: $\left|\left(\left(f_{n}, s\right)\right)\right|=\mid \sum_{k=1}^{N}$ $\int_{t_{k-1}}^{t_{k}}\left(f_{n}(s), v_{k}^{*}\right) d s \mid \leq \sum_{k=1}^{N}\left\|\int_{t_{k-1}}^{t_{k}} f_{n}(s) d s\right\|\left\|v_{k}^{*}\right\|_{*}$, where $\|\cdot\|_{*}$ denotes the norm in $X^{*}$. It follows that $\left|\left(\left(f_{n}, s\right)\right)\right| \leq\left\|f_{n}\right\|_{w} \sum_{k=1}^{N}\left\|v_{k}^{*}\right\|_{*} \rightarrow 0(n \rightarrow \infty)$, and the proof is complete.

Q.E.D.

We now turn to the question of existence of integral solutions to equation (2). The following conditions will be assumed throughout. $H(A): \quad A \subseteq D(A) \subset X \rightarrow 2^{X}$ is $m$-accretive and $-A$ generates a compact semigroup on $\overline{D(A)}$.
$H(F): \quad F: T \times \overline{D(A)} \rightarrow P_{w k c}(X)$ satisfies
(1) $t \rightarrow F(t, x)$ is measurable, for all $x \in X$,
(2) $x \rightarrow F(t, x)$ is $h$-continuous, a.e. $t \in T$,
(3) $|F(t, x)|=\sup \{\|v\|: v \in F(t, x)\} \leq \alpha(t)+\beta(t)\|x\|, \quad$ a.e. $\quad t \in T$, $\forall x \in \overline{D(A)}$, for some $\alpha, \beta \in L^{p}\left(T, \mathbf{R}_{+}\right), 1<p<\infty$.
$H(k): \quad k \in A C(T, \mathbf{R}), \dot{k} \in B V(T, \mathbf{R}), k(0)=1$,
$H(g): \quad g \in W^{1, p}(T, X), g(0) \in \overline{D(A)}$.

Let $S, S_{e} \subseteq C(T, X)$ denote the sets of integral solutions to equations $(\underline{\underline{1}})$ and (2), respectively. As a consequence of $[2$, theorem 3.2], if conditions $H(A), H(F), H(k)$ and $H(g)$ 
hold, then $S$ is nonempty. The nonemptiness of $S_{e}$ is established next.

Theorem 3.2: $\quad$ Let assumptions $H(A), H(F), H(k)$, and $H(g)$ be satisfied. If also $X^{*}$ is uniformly convex, then $S_{e} \neq \emptyset$.

Proof: We first derive an a priori bound for elements in $S$ (and in particular for elements of $S_{e}$, since $\left.S_{e} \subseteq S\right)$. Let $x \in S$, and let $y \in C(T, X)$ be the unique integral solution [7] of the Cauchy problem

$$
\dot{y}(t)+A y(t) \ni 0(t \in T), x(0)=x_{0},
$$

where $x_{0}$ is given by $(\underline{\underline{3}})(i i i)$ (see Section 2). By properties of integral solutions, we have

$$
\|x(t)-y(t)\| \leq \int_{0}^{t}\|G(x)(s)-f(s)\| d s,(t \in T)
$$

where $f(t) \in F(t, x(t))$ a.e. and $G$ is defined by $(\underline{3})$. Hence, invoking $(\underline{\underline{3}})$ and $H(F)(3)$, we obtain

$$
\begin{aligned}
\|x(t)\| \leq\|y\|_{\infty} & +\int_{0}^{t}\left(\|h(s)\|+\|r * h\|_{\infty}+|r(0)|\|x(s)\|+|r(t)|\left\|x_{0}\right\|\right. \\
& +\|x * \dot{r}(s)\|) d s+\int_{0}^{t}(\alpha(s)+\beta(s)\|x(s)\|) d s
\end{aligned}
$$

where $\|\cdot\|_{\infty}$ denotes the norm in $L^{\infty}(T, X)$.

Let $\gamma(s)=|r(0)|+\operatorname{var}(r:[0, s])$ and $x_{s}(\tau)=x(s-\tau), \tau \in[0, s]$. It follows that there exists $c>0$ such that

$$
\left\|x_{t}\right\|_{\infty} \leq c+\int_{0}^{t} \xi(s)\left\|x_{s}\right\|_{\infty} d s
$$

where $\xi(s)=\gamma(s)+\beta(s) \in L^{p}\left(T, \mathbb{R}_{+}\right)$(cf. $\left.H(F)(3), H(g)\right)$. This implies, by Gronwall's inequality, that

$$
\|x(t)\| \leq M, \forall t \in T, \forall x \in S,
$$

for some $M>0$. Using again (3) in conjunction with $H(F)(3)$ and $H(g)$, we see that there is $\psi \in L^{p}\left(T, \boldsymbol{R}_{+}\right)$such that

$$
\|G(x)(t)\|+|F(t, x(t))| \leq \psi(t), \text { a.e. on } T
$$


and we may assume without loss of generality that this inequality holds for all $x \in C(T, \overline{D(A)})$. (Otherwise, we replace $G(x)(\cdot)$ and $F\left(\cdot, x(\cdot)\right.$ ) by $G\left(p_{M}(x)\right)(\cdot)$ and $F\left(\cdot, p_{M}(x(\cdot))\right)$, respectively, where $p_{M}$ is the $M$-radial retraction, in what follows).

Next set $K=\left\{v \in L^{p}(T, X):\|v(t)\| \leq \psi(t)\right.$, a.e. $\}$ and let $\theta: L^{1}(T, X) \rightarrow C(T, X)$ be the map that assigns to each $v \in L^{1}(T, X)$ the unique integral solution of $\dot{x}(t)+A x(t) \ni v(t)$, $x(0)=x_{0}$. Since $K$ is bounded in $L^{p}(T, X)$, we may invoke [4, theorem 1] to conclude that

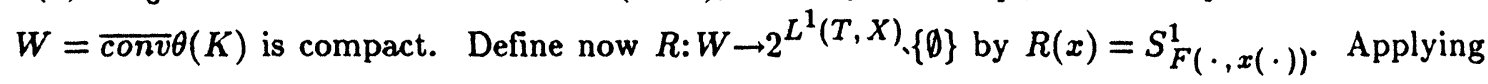
theorem 1.1 of [21], we can find a continuous map $\varphi: W \rightarrow L_{w}(T, X)$, such that $\varphi(x) \in \operatorname{ext} R(x)$ $\forall x \in W$. According to Benamara [6], ext $R(x)=\operatorname{ext} S_{F(\cdot, x(\cdot))}^{1}=S_{\text {ext } F(\cdot, x(\cdot))}^{1}$. Finally, let $u=\theta o(G-\varphi): W \rightarrow W$ (remark that $(G-\varphi)(w) \subseteq K$ ), and recall that by [24, corollary 2.3.1, p. 67] $\theta$ is sequentially continuous from $K \subseteq L^{p}(T, X)$ with the weak topology into $C(T, X)$ and $G(\cdot)$ as given by $(\underline{3})$ is linear, continuous from $C(T, X)$ to $L^{p}(T, X)$. This and the continuity of $\varphi$ from $W$ to $L_{w}(T, X)$, in conjunction with Lemma 3.1 imply that $u$ is continuous in the $C(T, X)$ topology. Since $W$ is compact in $C(T, X)$, we can apply Schauder's fixed point theorem to obtain $x \in W$ such that $x=u(x)$. Obviously $x$ is an integral solution to equation $(\underline{\underline{2}})$, so that $S_{e} \neq \emptyset$, as claimed.

Q.E.D.

\section{DENSITY OF EXTREMAL SOLUTIONS}

In this section, we show that $S_{e}$ is dense in $S$ for the $C(T, X)$-topology. This supplements the relaxation result obtained by the authors in [2] (see [2, theorem 4.1]). Note that the multifunction $(t, x) \rightarrow \operatorname{ext} F(t, x)$ is not necessarily closed valued or lower semicontinuous, let alone Hausdorff-Lipschitz, so that the assumption on the orientor field in theorem 4.1 of [2] is generally violated. Consequently, the next theorem is a genuine new approximation result.

We need the following stronger hypothesis on the orientor field $F(t, x)$.

$\underline{H(F)^{\prime}:} \quad F: T \times X \rightarrow P_{w k c}(X)$ satisfies

(1) $t \rightarrow F(t, x)$ is measurable, $\forall x \in X$,

(2) $\quad h(F(t, x), F(t, y)) \leq l(t)\|x-y\| \quad \forall x, y \in X$, a.e. $\quad$ on $\quad T$, for some $l \in L^{1}\left(T, R_{+}\right)$,

(3) there exist $\alpha, \beta \in L^{p}\left(T, \boldsymbol{R}_{+}\right), \quad 1<p<\infty$, such that $|F(t, x)|=$ $\sup \{\|v\|: v \in F(t, x)\} \leq \alpha(t)+\beta(t)\|x\|$ a.e. on $T$. 
Theorem 4.1: If $X^{*}$ is uniformly convex and assumptions $H(A), H(F)^{\prime}, H(k)$ and $H(g)$ are satisfied, then $S={\overline{S_{e}}}^{C(T, X)}$.

Proof: Let $x(\cdot) \in S$. Then $x$ is an integral solution of

$$
\left\{\begin{array}{c}
\dot{x}(t)+A x(t)=G(x)(t)-f(t), \text { a.e. on } T, \\
x(0)=x_{0}
\end{array}\right\}
$$

with $f \in L^{1}(T, X), f(t) \in F(t, x(t))$, a.e. Let $W \subseteq C(T, X)$ be as in the proof of theorem 3.2. Given $y \in W$ and $\epsilon>0$, let $H: T \rightarrow 2^{X},\{\emptyset\}$ be defined by

$$
H(t)=\left\{u \in X:\|f(t)-u\|<\frac{\epsilon}{2 M b}+d(f(t), F(t, y(t))), u \in F(t, y(t))\right\}
$$

where $M>0$ is the a priori bound for elements of $S$ obtained in the proof of theorem 3.2. We then have

$$
G r H=\left\{(t, u) \in G r F(\cdot, y(\cdot)):\|f(t)-u\|<\frac{\epsilon}{2 M b}+d(f(t), F(t, y(t)))\right\} .
$$

Using condition $H(F)^{\prime},(1)$ and (2) and [19, theorem 3.3], we infer that $\operatorname{Gr} F(\cdot, y(\cdot)) \in B(T) \times$ $B(X)$, where $B(T)$ (resp. $B(X)$ ) denotes the Borel $\sigma$-field of $T$ (resp. of $X$ ). Apply Aumann's selection theorem (see Wagner [25, theorem 5.10]), to obtain a measurable map $u: T \rightarrow X$ such that $u(t) \in H(t)$, a.e. on $T$. Thus if we define $\Phi: W \rightarrow 2^{L^{1}(T, X)}$ by

$$
\Phi(y)=\left\{u \in S_{F(\cdot, y(\cdot))}^{1}:\|f(t)-u(t)\|<\frac{\epsilon}{2 M b}+d(f(t), F(t, y(t))) \text { a.e. }\right\},
$$

it follows that $\Phi$ has nonempty decomposable values. In addition by [13, proposition 2.3], $y \rightarrow \Phi(y)$ is l.s.c. Therefore $y \rightarrow \overline{\Phi(y)}$ is l.s.c., with nonempty, closed decomposable values. So, we can apply Fryzkowski's selection theorem [13] and get a continuous map $u_{\epsilon}: W \rightarrow L^{1}(T, X)$ such that $u_{\epsilon}(y) \in \overline{\Phi(y)}$ for all $y \in W$. Then we have:

$$
\begin{array}{r}
\left\|f(t)-u_{\epsilon}(y)(t)\right\| \leq \frac{\epsilon}{2 M b}+d(f(t), F(t, y(t))) \\
\leq \frac{\epsilon}{2 M b}+l(t)\|x(t)-y(t)\|, \text { a.e. on } T .
\end{array}
$$

Now use theorem 1.1 in [21] to obtain a continuous mapping $v_{\epsilon}: W \rightarrow L_{w}(T, X)$, such that $v_{\epsilon}(y) \in \operatorname{ext} S_{F(\cdot, y(\cdot))}^{1}=S_{e x t F(\cdot y(\cdot))}^{1}$ and $\left\|u_{\epsilon}(y)-v_{\epsilon}(y)\right\|_{w}<\epsilon$ for all $y \in W$.

Next let $\epsilon_{n} \downarrow 0$, let $u_{n}=u_{\epsilon_{n}}, v_{n}=v_{\epsilon_{n}}$ be as above and let $x_{n} \in W$ satisfy $x_{n}=\theta o\left(G-v_{n}\right)\left(x_{n}\right)$. (Here $\theta$ is the solution map introduced in the proof of theorem 3.2, and the existence of $x_{n}$ follows from Schauder's fixed point theorem. Note that $x_{n} \in S_{e}$ ). Since $W$ is compact in $C(T, X)$ (cf. the proof of theorem 3.2), we may assume that $x_{n} \rightarrow \bar{x}$ in $C(T, X)$, 
as $n \rightarrow \infty$. Invoking Benilan's inequality [7], we also have

$$
\left\|x(t)-x_{n}(t)\right\|^{2} \leq 2 \int_{0}^{t}\left(J\left(x(s)-x_{n}(s), G(x)(s)-f(s)-G\left(x_{n}\right)(s)+v_{n}\left(x_{n}\right)(s)\right) d s\right.
$$

where $(\cdot, \cdot)$ denotes the duality pairing between $X^{*}$ and $X$, and $J$ stands for the duality map of $X$. (Note that $J$ is single-valued and uniformly continuous, since $X^{*}$ is uniformly convex). It follows that

$$
\begin{aligned}
& \left\|x(t)-x_{n}(t)\right\|^{2} \leq 2 \int_{0}^{t}\left(J\left(x(s)-x_{n}(s)\right), G(x)(s)-G\left(x_{n}\right)(s)\right) d s \\
& +2 \int_{0}^{t}\left(J\left(x(s)-x_{n}(s)\right), v_{n}\left(x_{n}\right)(s)-u_{n}\left(x_{n}\right)(s)\right) d s \\
& +2 \int_{0}^{t}\left(J\left(x(s)-x_{n}(s)\right), u_{n}\left(x_{n}\right)(s)-f(s)\right) d s .
\end{aligned}
$$

Using ( $\underline{\underline{3}})$ we see that

$$
\begin{gathered}
\int_{0}^{t}\left(J\left(x(s)-x_{n}(s)\right), G(x)(s)-G\left(x_{n}\right)(s)\right) d s \\
\left.\leq \int_{0}^{t} \| x(s)-x_{n}(s)\right)\|\gamma(s)\| x_{s}-\left(x_{n}\right)_{s} \|_{\infty} d s \\
\leq \int_{0}^{t} \gamma(s)\left\|x_{s}-\left(x_{n}\right)_{s}\right\|_{\infty}^{2} d s,
\end{gathered}
$$

where $\gamma(s)=|r(0)|+\operatorname{var}(r ;[0, s])\left(\gamma \in L^{\infty}\left(T, \mathbb{R}_{+}\right)\right)$, and $x_{s}(\tau)=x(s-\tau), \tau \in[0, s]$

Also remark that by construction $v_{n}\left(x_{n}\right)-u_{n}\left(x_{n}\right) \rightarrow \|^{\prime} w_{0}$ and $\left\{v_{n}\left(x_{n}\right)-u_{n}\left(x_{n}\right)\right\}_{n \geq 1}$ is a bounded subset of $L^{p}(T, X)$ (cf. $H\left(F^{\prime}\right)(3)$ and the proof of theorem 3.2). Applying lemma 3.1 implies then $v_{n}\left(x_{n}\right)-u_{n}\left(x_{n}\right) \rightarrow 0$, weakly in $L^{p}(T, X)$. Furthermore, inasmuch as $x_{n} \rightarrow \bar{x}$ in $C(T, X)$ and $J$ is uniformly continuous from $X$ to $X^{*}$, we infer that

$$
\int_{0}^{t}\left(J\left(x(s)-x_{n}(s)\right), v_{n}\left(x_{n}\right)(s)-u_{n}\left(x_{n}\right)(s)\right) d s \rightarrow 0 \quad(n \rightarrow \infty) .
$$

Finally, we have 


$$
\begin{gathered}
\int_{0}^{t}\left(J\left(x(s)-x_{n}(s)\right), u_{n}\left(x_{n}\right)(s)-f(s)\right) d s \\
\leq \int_{0}^{t}\left\|x(s)-x_{n}(s)\right\|\left(\frac{\epsilon_{n}}{2 M b}+l(s)\left\|x_{n}(s)-x(s)\right\|\right) d s \\
\leq \epsilon_{n}+\int_{0}^{t} l(s)\left\|x_{s}-\left(x_{n}\right)_{s}\right\|_{\infty}^{2} d s .
\end{gathered}
$$

Therefore, passage to the limit in (岂), as $n \rightarrow \infty$, yields

$$
\left\|x_{t}-\bar{x}_{t}\right\|_{\infty}^{2} \leq 2 \int_{0}^{t}(\gamma(s)+l(s))\left\|x_{s}-\bar{x}_{s}\right\|_{\infty}^{2} d s
$$

Apply Gronwall's inequality to conclude that $x=\bar{x}$. Since $x_{n} \in S_{e}$ and $x_{n} \rightarrow \bar{x}=x$ in $C(T, X)$, it follows that $S \subseteq{\overline{S_{e}}}^{C(T, X)}$. (Recall that $S_{e} \subseteq S$ ). But from the remark following theorem 4.1 in [2], we know that $S$ is compact in $C(T, X)$. This implies that $S={\overline{S_{e}}}^{C(T, X)}$, and the proof is complete.

$\underline{\underline{Q . E . D .}}$

To illustrate the applicability of theorem 4.1, we consider a multivalued integro-partial differential equation arising in the study of obstacle problems [23]. Let $Z$ be a bounded domain of $\mathbb{R}^{N}$ with smooth boundary $\Gamma$. We consider the problem

$x(t, z)-\int_{0}^{t}\left(k(t-s)\left(\sum_{|\alpha| \leq m}(-1)^{|\alpha|} D^{\alpha} A_{\alpha}(z, \eta(x(s, z)))+F(s, z, x(s, z))\right) d s \ni g(t, z)\right.$, on $T \times Z$

$$
\left.D^{\gamma} x\right|_{T \times \Gamma}=0 \text { for all }|\gamma| \leq m-1 .
$$

where $k: T \rightarrow \mathbb{R}, g: T \times Z \rightarrow \mathbb{R}, \alpha=\left(\alpha_{1}, \ldots, \alpha_{N}\right),|\alpha|=\sum_{i=1}^{N} \alpha_{i}, D^{\alpha}=D_{1}^{\alpha_{1}}, \ldots, D_{N}^{\alpha}$ and $\eta(x)=$ $\left(D^{\beta} x\right)_{|\beta| \leq m}$ while $A_{\alpha}: Z \times \mathbb{R}^{N_{m} \rightarrow \mathbb{R}}\left(N_{m}=\frac{(N+m) !}{N ! m !}\right)$ and $F: T \times Z \times \mathbb{R} \rightarrow 2^{\mathbb{R}}$ satisfy the following conditions:

$\underline{H(A)^{\prime}:} \quad$ (1) $\quad z \rightarrow A_{\alpha}(z, \eta)$ is measurable, for all $\eta \in \mathbb{R}^{N_{m}}$,

(2) $\eta \rightarrow A_{\alpha}(z, \eta)$ is continuous, for a.a. $z \in \mathbb{R}$,

(3) $\quad\left|A_{\alpha}(z, \eta)\right| \leq a(z)+\|\eta\|_{R}^{p} \bar{N}_{m}^{-1}, 2 \leq p<\infty$, for some $a \in L^{q}(Z), \frac{1}{p}+\frac{1}{q}=1$,

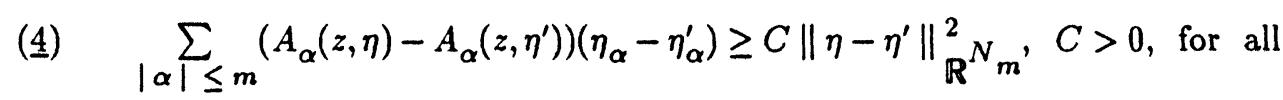




$$
\eta, \eta^{\prime} \in \mathbb{R}^{N_{m}}
$$

and respectively

$\underline{H(F)^{\prime \prime}:} \quad F(t, z, x)=\left\{v \in \mathbb{R}: f_{1}(t, z, x) \leq v \leq f_{2}(t, z, x)\right\}$, where $f_{i}: T \times Z \times \mathbb{R} \rightarrow \mathbb{R}, \quad(i=1,2)$ are given functions such that $(t, z) \rightarrow f_{i}(t, z, x)$ is measurable, $\left|f_{i}(t, z, x)-f_{i}(t, z, y)\right| \leq k(t, z)|x-y|$ a.e. with $k \in L^{1}\left(T \times Z, \mathbb{R}_{+}\right)$and $\left|f_{i}(t, z, x)\right| \leq \beta_{1}(t, z)+\beta_{2}(t, z)|x| \quad$ a.e. $\quad$ with $\quad \beta_{1} \in L^{2}\left(T \times Z, \mathbb{R}_{+}\right)$, $\beta_{2} \in L^{\infty}\left(T \times Z, \mathbb{R}_{+}\right)$.

Let $b: W_{0}^{m, p}(Z) \times W_{0}^{m, p}(Z) \rightarrow R$ be the Dirichlet form

$$
b(x, y)=\int_{Z} \sum_{|\alpha| \leq m} A_{\alpha}(z, \eta(x(z))) D^{\alpha} y(z) d z, \forall x, y \in W_{0}^{m, p}(Z),
$$

and define $\widehat{A}: W_{0}^{m, p}(Z) \rightarrow W^{-m, q}(Z)$ by

$$
\langle\hat{A} x, y\rangle=b(x, y), \forall x, y \in W_{0}^{m, p}(Z),
$$

where $\langle\cdot, \cdot\rangle$ denote the duality brackets between $W_{0}^{m, p}(Z)$ and $\left.W^{-m, q}(Z)\right)$. This definition makes sense in view of $H\left(A^{\prime}\right)(1),(2)$ and (3). Now let $X=L^{2}(Z)$ and define the "part" of $\widehat{A}$ in $X$ by $A(x)=\hat{A}(x)$, with $D(A)=\left\{x \in W_{0}^{m, p}(Z): \hat{A} x \in X\right\}$. On account of $H\left(A^{\prime}\right)(4)$, it follows that $A$ is maximal monotone (equivalently, $m$-accretive) in $X$. Moreover, making use of [23] (Example 6.1 and theorem 6.3, pp. 233-234), we conclude that $-A$ generates a compact semigroup on $X$.

In other words, assumption $H(A)$ is satisfied.

Next define $\widehat{F}: T \times X \rightarrow P_{f c}(X)$ by

$$
\hat{F}(t, x)=\left\{v \in X: f_{1}(t, z, x(z)) \leq v(z) \leq f_{2}(t, z, x(z)) \text { a.e. }\right\}
$$

Using $H(F)^{\prime \prime}$, it is easily seen that the multifunction $\hat{F}$ satisfies condition $H(F)^{\prime}$.

$\Rightarrow$ Finally assume that $g$ satisfies

$\underline{H(g)^{\prime}:} \quad g \in W^{1, p}\left(T, L^{2}(Z)\right)$,

and define $\hat{g}: T \rightarrow X$ by $\hat{g}(t)(\cdot)=g(t, \cdot)$. We then rewrite problem $(\underline{\underline{5}})$ in the abstract form

$$
x(t)+\int_{0}^{t} k(t-s)(A x(s)+\hat{F}(s, x(s))) d s \ni \hat{g}(t), t \in T .
$$

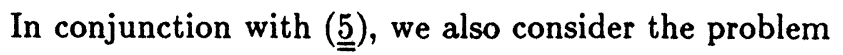




$$
\begin{gathered}
x(t, z)+\int_{0}^{t}\left(k ( t - s ) \left(\sum_{|\alpha| \leq m}(-1)^{|\alpha|} D^{\alpha} A_{\alpha}(z, \eta(x(s, z)))+\right.\right. \\
\left.+\left\{f_{1}(s, z, x(s, z)), f_{2}(s, z, x(s, z))\right\}\right) d s \ni g(t, z) \text { on } T \times Z \\
\left.D^{\gamma} x\right|_{T \times \Gamma}=0,|\gamma| \leq m-1
\end{gathered}
$$

whose abstract form is

$$
x(t)+\int_{0}^{t} k(t-s)(A x(s)+e x t \hat{F}(s, x(s))) d s \ni \widehat{g}(t), t \in T .
$$

We will say that $x \in C\left(T, L^{2}(Z)\right)$ is a solution of $(\underline{\underline{5}})$ (resp. $\left.(\underline{\underline{5}})_{e}\right)$ if $x$ is an integral solution of $(\underline{\underline{5}})^{\prime}\left(\operatorname{resp}(\underline{\underline{5}})_{e}^{\prime}\right)$. A direct application of theorem 4.1 now yields:

Theorem 4.2: Let assumptions $H(A)^{\prime}, H(F)^{\prime \prime}, H(g)^{\prime}$ and $H(k)$ be satisfied. If $x \in C\left(T, L^{2}(Z)\right)$ is a solution of $(\underline{5})$ and $\epsilon>0$, then there exists a solution $y \in C\left(T, L^{2}(Z)\right)$ of $(\underline{5})_{e}$ such that $\sup _{t \in T}\left(\int_{Z}|x(t, z)-y(t, z)|^{2} d z\right)^{1 / 2}<\epsilon$.

Remark: Invoking Barbu [5, theorem 2.6, p. 140], we can deduce that the functions $x$ and $y$ of theorem 4.2 are actually "strong" solutions of problems $(\underline{\underline{5}})$ and $(\underline{\underline{5}})^{\prime}$, respectively, satisfying $x, y \in C\left(T, L^{2}(Z) \cap L^{p}\left(T, W_{0}^{m, p}(Z)\right)\right.$ and $\dot{x}, \dot{y} \in L^{q}\left(T, W^{-m, q}(Z)\right)$.

\section{INFINITIE DIMENSIONAL CONTROL SYSTEMS}

In this section we use theorems 3.1 and 4.1 to derive a "bang-bang" theorem for a class of infinite dimensional nonlinear control systems. Specifically, we consider the problems

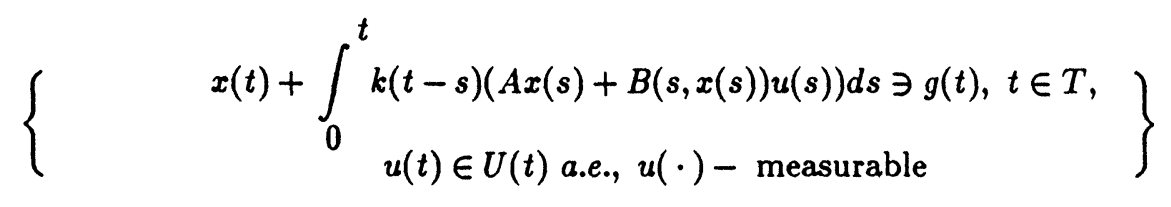

and

$$
\left\{\begin{array}{c}
x(t)+\int_{0}^{t} k(t-s)(A x(s)+B(s, x(s)) u(s)) d s \ni g(t), t \in T, \\
u(t) \in \operatorname{ext} U(t) \text { a.e., } u(\cdot)-\text { measurable. }
\end{array}\right\}
$$

where $k: T \rightarrow \mathbb{R}, g: T \rightarrow X, A: D(A) \subseteq X \rightarrow X$, while $B$ and $U$ satisfy the following conditions. (We denote by $Y$ a real separable Banach space modelling the control space).

$\underline{H(B):} \quad B: T \times X \rightarrow \mathcal{L}(Y, X)$ is a map such that 
(1) $t \rightarrow B(t, x) u$ is measurable,

(2) $\quad\left\|B(t, x)-B\left(t, x^{\prime}\right)\right\|_{\mathcal{L}} \leq l(t)\left\|x-x^{\prime}\right\|$ a.e. with $l(\cdot) \in L^{1}\left(T, \mathbb{R}_{+}\right)$,

(3) $\quad\|B(t, x)\|_{\mathcal{L}} \leq \alpha(t)+\beta(t)\|x\|$ a.e. with $\alpha, \beta \in L^{p}\left(T, \mathbb{R}_{+}\right), 1<p<\infty$.

$\underline{H(U):} \quad U: T \rightarrow P_{w k c}(Y)$ is a measurable multifunction such that $U(t) \subseteq V$, a.e. where $V$ is a fixed element of $P_{w k c}(Y)$.

Let $S, S_{e} \subseteq C(T, X)$ be the sets of integral solutions of equations $(\underline{\underline{6}})$ and $(\underline{\underline{6}})^{\prime}$, respectively, for any admissible control function. Also let $R(t)$ and $R_{e}(t)$ denote the corresponding reachable sets at time $t \in T$; i.e. $R(t)=\{x(t): x \in S\}$ and $R_{e}(t)=\left\{x(t): x \in S_{e}\right\}$.

Remark: The nonemptiness of $S, S_{e}, R(t)$ and $R_{e}(t)$ will be a consequence of theorem 3.2 in [2] and theorem 3.2 in Section 3 , as soon as equations $(\underline{\underline{6}})$ and $(\underline{\underline{6}})^{\prime}$ will be written in the abstract form $(\underline{\underline{1}})$ and $(\underline{\underline{2}})$, respectively. See the proof of theorem 5.1 below.

Theorem 5.1: If $X^{*}$ is uniformly convex and hypotheses $H(A), H(B), H(U)$, $H(k), H(g)$ are satisfied, then $S={\overline{S_{e}}}^{C(T, X)}$ and for every $t \in T, R(t)=\overline{R_{e}(t)}$.

Proof: Set $F(t, x)=B(t, x) U(t)=U\{B(t, x) u: u \in U(t)\} \in P_{w k c}(X)$. Let $v \in X$ and let $u_{n}: T \rightarrow Y, n \geq 1$ be measurable functions such that $U(t)={\overline{\left\{u_{n}(t)\right\}_{n}}}_{n 1}$ (in $Y$ ). Note that the existence of $u_{n}$ is a consequence of hypothesis $H(U)$ and theorem 4.2 in [25]. One has

$$
\begin{gathered}
d(v, F(t, x))=\inf _{n \geq 1}\left\|v-B(t, x) u_{n}(t)\right\| \\
\Rightarrow d(v, F(t, x)) \text { is measurable } \\
\Rightarrow t \rightarrow F(t, x) \text { is measurable. }
\end{gathered}
$$

Let now $x, x^{\prime} \in X$ and $v \in F(t, x)$. Then $v=B(t, x) u$, with $u \in U(t)$. In view of $H(B)(2)$ and $H(U)$, we get

$$
d\left(v, F\left(t, x^{\prime}\right)\right) \leq\left\|B(t, x) u-B\left(t, x^{\prime}\right) u\right\| \leq l(t)|V|\left\|x-x^{\prime}\right\|,
$$

with $|V|=\sup \left\{\|u\|_{Y}: u \in U(t)\right\}$. Therefore

$$
h\left(F(t, x), F\left(t, x^{\prime}\right)\right) \leq \hat{l}(t)\left\|x-x^{\prime}\right\|, \quad\left(\hat{l}(t)=l(t)|V| \in L^{1}\left(T, \mathbb{R}_{+}\right)\right) .
$$

Finally, because of $H(B)(\underline{3})$, we have

$$
|F(t, x)|=\sup \{\|z\|: z \in F(t, x)\} \leq \hat{\alpha}(t)+\hat{\beta}(t)\|x\| \text {, a.e. on } T,
$$

where $\hat{\alpha}(t)=\alpha(t)|V|$ and $\hat{\beta}(t)=\beta(t)|V|$, so that $\hat{\alpha}, \hat{\beta} \in L^{p}\left(T, \mathbb{R}_{+}\right)$. It follows that problems $(\underline{\underline{6}})$ (resp. $\left.(\underline{\underline{6}})^{\prime}\right)$ can be rewritten in the form $(\underline{\underline{1}})$ (resp. (르)) in $X$. An application of 
theorem 4.1 then leads to the desired conclusions.

$\underline{\underline{Q . E . D .}}$

In theorem 5.1, we may also assume that $Y$ is the dual of a separable Banach space Yै, i.e. $Y=\mathrm{q}^{*}$. In this case $H(U)$ changes to $H(U)^{\prime}: \quad U(t)=U,(t \in T)$ is a nonempty, $w^{*}$-compact and convex subset of $Y$, while the measurability of the admissible control functions is viewed as $w^{*}$ measurability. (Here " $w^{* n}$ stands for "weak star"). Minor changes in the proof of theorem 5.1 lead us to the following result.

Theorem 5.2: If $X^{*}$ is uniformly convex and assumptions $H(A), H(B), H(U)^{\prime}$, $H(k), H(g)$ are satisfied, then the conclusions of theorem 5.1 hold.

We will apply theorem 5.2 to obtain a "bang-bang" principle for a controlled integral equation arising in the study of materials with memory [10]. As in Section 4, we let $Z$ be a bounded domain of $\mathbb{R}^{N}$ with smooth boundary $\Gamma$. Consider the problem

$$
\begin{gathered}
x(t, z)-\int_{0}^{t}\left(k(t-s)\left(\sum_{i=1}^{N} \frac{\partial}{\partial z_{i}}\left|\frac{\partial x}{\partial z_{i}}\right|^{p-2} \frac{\partial x}{\partial z_{i}}\right)+c(s, z, x(s, z)) u(s, z)\right) d s, \quad \ni g(t, z) \text { on } T \times Z . \\
\left.x\right|_{T \times \Gamma}=0,|u(t, z)| \leq \mu, u(\cdot, \cdot) \text {-measurable, }
\end{gathered}
$$

where $2 \leq p<\infty, \quad \mu>0, \quad k: T \rightarrow \mathbb{R}, \quad g: T \times Z \rightarrow \mathbb{R}$, and $c: T \times Z \times \mathbb{R} \rightarrow \mathbb{R}$. We assume that assumptions $H(k)$ and $H(g)^{\prime}$ (see Section 4) hold. We also impose the following restrictions on the function $c$ :

$\underline{H(c):} \quad(\underline{1}) \quad(t, z) \rightarrow c(t, z, x)$ is measurable,

(2) $\left|c(t, z, x)-c\left(t, z, x^{\prime}\right)\right| \leq l(t, z)\left|x-x^{\prime}\right|$ a.e., with $l \in L^{1}\left(T \times Z, \mathbb{R}_{+}\right)$,

(3) $|c(t, z, x)| \leq \alpha(t, z)+\beta(t, z)|x|$ a.e., with $\alpha \in L^{p}\left(T \times Z, \mathbb{R}_{+}\right)$, $\beta \in L^{\infty}\left(T \times Z, \mathbb{R}_{+}\right)$.

We again choose $X=L^{2}(Z)$, and define $A$ to be the pseudo-Laplacian, i.e. $A x=-\sum_{i=1}^{N} \frac{\partial}{\partial z_{i}}\left(\left|\frac{\partial x}{\partial z_{i}}\right|^{p-2} \frac{\partial x}{\partial z_{i}}\right)$ with $D(A)=\left\{x \in W_{0}^{1, p}(Z): A x \in X\right\}$. It is well known (see e.g. [24] Example 2.2.4 and Remark 2.2.5, pp. 59-60) that $A$ is cyclically maximal monotone in $X$ and $-A$ generates a compact semigroup on $X$. Therefore $A$ satisfies $H(A)$. Next define $\widehat{c}: T \times X \rightarrow X$ by $\widehat{c}(t, x)(z)=c(t, z, x(z))$, and $\widehat{g}: T \rightarrow X$ by $\widehat{g}(t)(\cdot)=g(t, \cdot)$. Also, set $Y=L^{\infty}(Z)$ and $U=\left\{u \in L^{\infty}(Z):|u(z)| \leq \mu\right.$, a.e. $\}$. From [15, p. 79] it follows that extU $=\left\{u \in L^{\infty}(Z): \lambda[z \in Z:|u(z)| \neq \mu]=0\right\}$, where $\lambda$ stands for the Lebesgue measure on 
$\mathbb{R}^{N}$

Rewriting problem (ㄱ) in $X$ as

$$
\begin{gathered}
x(t)+\int_{0}^{t} k(t-s)(A x(s)+\hat{c}(s, x(s)) u(s)) d s \ni \hat{g}(t), \text { on } T \\
u(t) \in U, \text { a.e, } u=w^{*} \text {-measurable. }
\end{gathered}
$$

it is readily seen that all of the assumptions of theorem 5.2 are satisfied, provided that we take $B(t, x) u=\widehat{c}(t, x) u$. (Note that $H(c)$ then implies $H(B)$ ). Applying theorem 5.2 we finally obtain

Theorem 5.3: If conditions $H(c), H(k), H(g)$ are satisfied, $x$ is a trajectory of $(\underline{\underline{7}})$ and $\epsilon>0$ is given, then there exists a control $v \in U$ such that $\lambda[z \in Z:|v(z)| \neq \mu]=0$, which generates a trajectory $y \in C\left(T, L^{2}(Z)\right)$ of $(\underline{\underline{7}})$ with the property that

$$
\sup _{t \in T} \int_{Z}|y(t, z)-x(t, z)|^{2} d z<\epsilon .
$$

Remark: Here the term “trajectory” designates an integral solution to $(\underline{\underline{7}})$ '. Since $A$ is cyclically maximal monotone in $X$, it actually follows that $x$ and $y$ in theorem 5.3 are "strong" solutions of problem (7). See e.g. [24, p. 28 and 42].

\section{REFERENCES}

[1] S. Aizicovici, "An integrodifferential equation with a discontinuous nonlinearity", $A n$. St. Univ. “Al. I. Cuza”, Iasi 26, (1980), pp. 353-360.

[2] S. Aizicovici and N.S. Papageorgiou, "Multivalued Volterra integral equations in Banach spaces", Funkcialaj Ekvacioj - to appear.

[3] E. Avgerinos and N.S. Papageorgiou, "Optimal control and relaxation for a class of nonlinear distributed parameter systems", Osaka J. Math. 27, (1990), pp. 745-767.

[4] P. Baras, "Compacité de l'opérateur $f \rightarrow u$ solution d'une équation non-linéaire $\frac{d u}{d t}+A u \ni f^{\prime \prime}, C R A S$ Paris, $t$ 286, (1978), pp. 1113-1116.

[5] V. Barbu, "Nonlinear Semigroups and Differential Equations in Banach Spaces", Noordhoff International Publishing, Leiden, The Netherlands (1976).

[6] M. Benamara, "Points Extrémaux, Multi-applications et Fonctionelles Intégrales", Thèse du 3ème cycle, Université de Grenoble (1975).

[7] Ph. Bénilan, “Equations d'Evolution dans un Espace de Banach Quelconque et 
Applications", Thèse, Université de Paris XI, Orsay (1972).

[8] H. Brézis, "Opérateurs Maximaux Monotones et Semigroupes de Contractions dans les Espaces de Hilbert", North Holland, Amsterdam (1973).

[9] K.C. Chang, "The obstacle problem and partial differential equations with discontinuous nonlinearities", Comm. Pure and Appl. Math. 33, (1980), pp. 117-146.

[10] B. Coleman and M. Gurtin, "Equi-presence and constitutive equations for rigid heat conductors", Z. Angew. Math. und Phys. 18, (1967), pp. 199-208.

[11] M.G. Crandall and J.A. Nohel, "An abstract functional differential equation and a related Volterra equation", Israel J. Math. 29, (1978), pp. 313-328.

[12] J. Diestel and J. Uhl, "Vector Measures", Math. Surveys, Vol. 15, AMS, Providence, RI (1977).

[13] A. Fryszkowski, "Continuous selections for a class of nonconvex multivalued maps", Studia Math. 78, (1983), pp. 163-174.

[14] S. Gutman, "Evolutions governed by m-accretive plus compact operators", Nonlinear Analysis-TMA 7, (1983), pp. 707-717.

[15] R. Holmes, "Geometric Functional Analysis and its Applications", Springer, Berlin (1975).

[16] T. Kiffe, "A perturbation of an abstract Volterra equation", SIAM J. Math. Anal. 11, (1980), pp. 1036-1046.

[17] E. Mitidieri and I. Vrabie, "Differential inclusions governed by nonconvex perturbations of $m$-accretive operators", Differential and Integral Equations 2, (1989), pp. 523-531.

[18] N.S. Papageorgiou, "Volterra integrodifferential inclusions in reflexive Banach spaces", Funkcialaj Ekvacioj 34, (1991), pp. 257-277.

[19] N.S. Papageorgiou, "On measurable multifunctions with applications to random multivalued equations", Math. Japonica 32, (1987), pp. 437-464.

[20] N.S. Papageorgiou, "Nonlinear Volterra integrodifferential evolution inclusions and optimal control", Kodai Math. Jour. 14, (1991), pp. 254-280.

[21] A. Tolstonogov, "Extreme continuous selectors of multivalued maps and the "bangbang” principle for evolution inclusions", Soviet Math. Dokl. 317, (1991), pp. 1-8.

[22] I. Vrabie, "Compactness methods for an abstract nonlinear Volterra integrodifferential equation", Nonlinear Analysis-TMA 5, (1981), pp. 355-371.

[23] I. Vrabie, "The nonlinear version of Pazy's local existence theorem", Israel J. Math. 32, (1979), pp. 221-235. 
[24] I. Vrabie, “Compactness Methods for Nonlinear Evolutions", Pitman, Boston (1987).

[25] D. Wagner, "Survey of measurable selection theorems", SIAM J. Control and Optim. 15, (1977), pp. 859-903. 


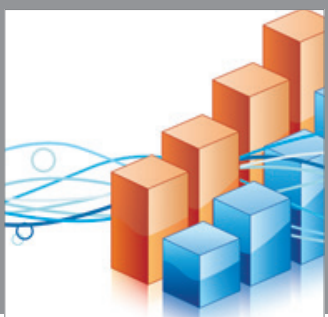

Advances in

Operations Research

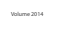

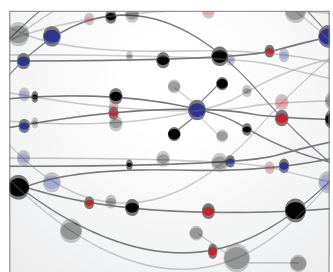

\section{The Scientific} World Journal
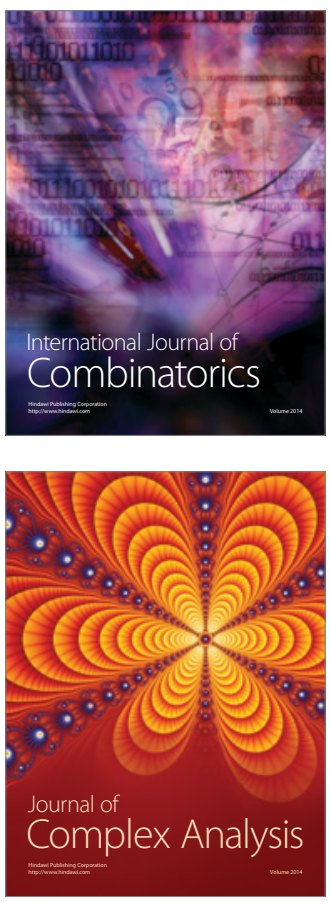

International Journal of

Mathematics and

Mathematical

Sciences
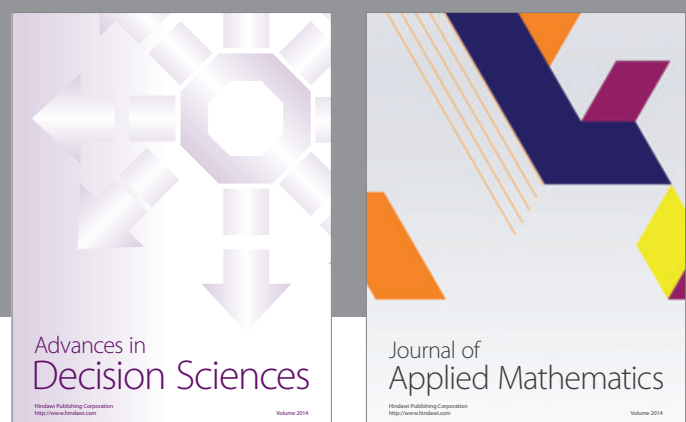

Journal of

Applied Mathematics
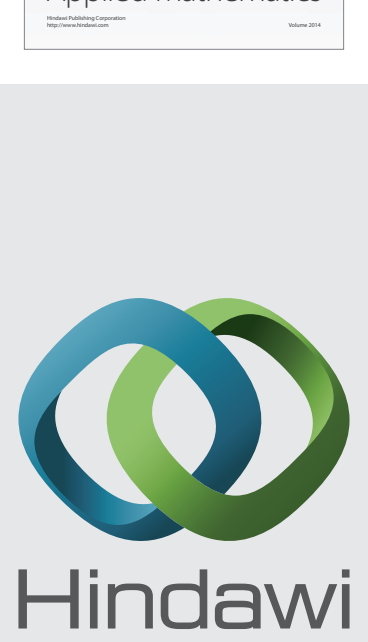

Submit your manuscripts at http://www.hindawi.com
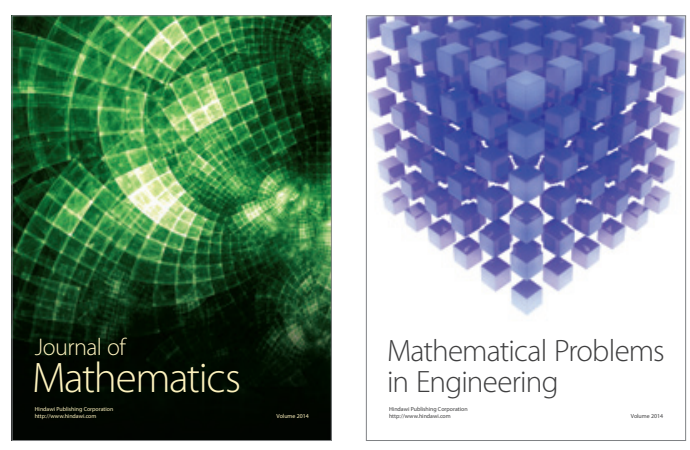

Mathematical Problems in Engineering
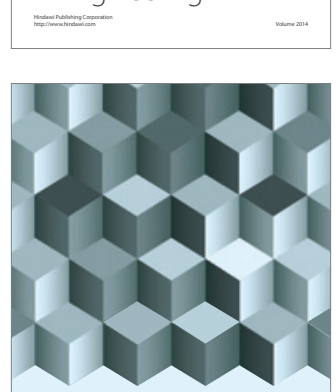

Journal of

Function Spaces
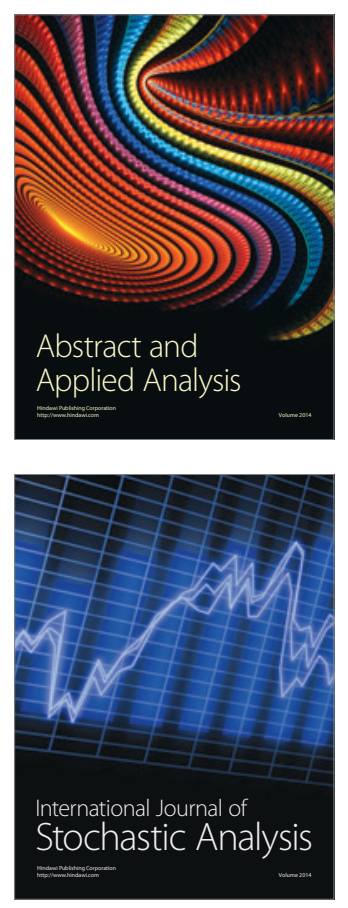

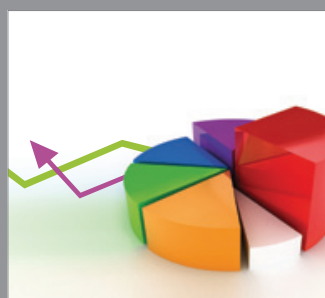

ournal of

Probability and Statistics

Promensencen
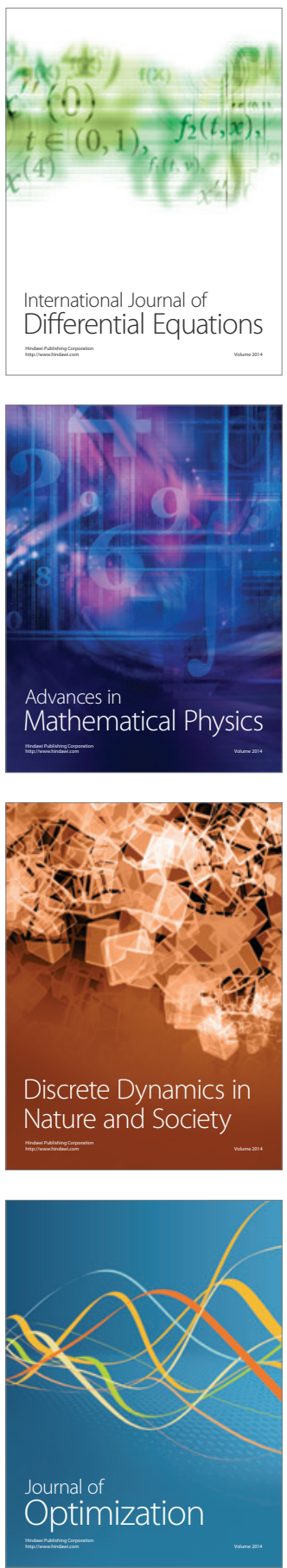